\title{
Social media networking in pediatric hydrocephalus: a point-prevalence analysis of utilization
}

\author{
Ghassan Awad Elkarim, BSc, ${ }^{1}$ Naif M. Alotaibi, MD, ${ }^{1-3}$ Nardin Samuel, PhD, ${ }^{4}$ \\ Shelly Wang, MD, MPH, ${ }^{1}$ George M. Ibrahim, MD, PhD, ${ }^{1}$ Aria Fallah, MD, MSc, ${ }^{5}$ \\ Alexander G. Weil, MD, ${ }^{6}$ and Abhaya V. Kulkarni, MD, PhD ${ }^{1,7}$
}

'Division of Neurosurgery, Department of Surgery; ${ }^{2}$ Institute of Medical Science, Faculty of Medicine; ${ }^{4} \mathrm{MD} / \mathrm{PhD}$ Program, University of Toronto; ${ }^{7}$ Division of Neurosurgery, Hospital for Sick Children, Toronto, Ontario; ${ }^{6}$ Division of Pediatric Neurosurgery, Department of Surgery, Sainte Justine Hospital, University of Montreal, Quebec, Canada; ${ }^{3}$ Department of Neurosurgery, National Neuroscience Institute, King Fahad Medical City, Riyadh, Saudi Arabia; and 5Department of Neurosurgery, David Geffen School of Medicine at University of California, Los Angeles, California

OBJECTIVE A recent survey has shown that caregivers of children with shunt-treated hydrocephalus frequently use social media networks for support and information gathering. The objective of this study is to describe and assess social media utilization among users interested in hydrocephalus.

METHODS Publicly accessible accounts and videos dedicated to the topic of hydrocephalus were comprehensively searched across 3 social media platforms (Facebook, Twitter, and YouTube) throughout March 2016. Summary statistics were calculated on standard metrics of social media popularity. A categorization framework to describe the purpose of pages, groups, accounts, channels, and videos was developed following the screening of 100 titles. Categorized data were analyzed using nonparametric tests for statistical significance.

RESULTS The authors' search identified 30 Facebook pages, 213 Facebook groups, 17 Twitter accounts, and 253 YouTube videos. These platforms were run by patients, caregivers, nonprofit foundations, and patient support groups. Most accounts were from the United States $(n=196)$, followed by the United Kingdom $(n=31)$, Canada $(n=17)$, India $(n=15)$, and Germany $(n=12)$. The earliest accounts were created in 2007, and a peak of 65 new accounts were created in 2011. The total number of users in Facebook pages exceeded those in Facebook groups $(p<0.001)$. The majority of users in Facebook groups were in private groups, in contrast to public groups $(p<0.001)$. The YouTube videos with the highest median number of views were for surgical products and treatment procedures.

CONCLUSIONS This study presents novel observations into the characteristics of social media use in the topic of hydrocephalus. Users interested in hydrocephalus seek privacy for support communications and are attracted to treatment procedure and surgical products videos. These findings provide insight into potential avenues of hydrocephalus outreach, support, or advocacy in social media.

https://thejns.org/doi/abs/10.3171/2017.3.PEDS16552

KEY WORDS children; hydrocephalus; internet; pediatric; social media network

$\mathrm{H}$ YDROCEPHALUS is a common pediatric neurosurgical condition, and many patients with treated hydrocephalus have chronic functional disability with impairments in cognition, ambulation, vision, speech, emotions, and dexterity. ${ }^{714}$ There are many socioeconomic factors associated with worse outcome for children with hydrocephalus, including total household income less than $\$ 100,000$, no parent with a university degree, and worse family functioning..${ }^{14}$ Social support has been shown to be beneficial for patients and caregivers dealing with chronic disease: it reduces the stress of having a child with a physical handicap on parent adjustment, and it improves the patient's adjustment in terms of community acceptance, developmental progress, and involvement in activities in the home and the community. ${ }^{4,6,10}$

Online social media networks (SMNs) are increasingly used by patients and family members to access support groups and gather educational information. ${ }^{2}$ Several stud- 
ies have reported positive impact from social media use on outcomes in obesity, depression, cancer, heart disease, stroke, and diabetes. ${ }^{8,13,20}$ Despite the increasing popularity and benefit of social media in health care, little is known about the utilization of social media in pediatric hydrocephalus.

In this report, we describe and analyze social media metrics related to hydrocephalus. The results can inform practicing physicians of the presence and magnitude of the online community interested in hydrocephalus. This information may also be valuable for interested patients wishing to access relevant online support groups.

\section{Methods}

\section{Search Strategy, Data Collection, and Extraction}

A comprehensive search on 3 social media platforms (Facebook, YouTube, and Twitter) was performed by 2 authors (G.A.E. and N.M.A.) using the term "hydrocephalus." The search and data collection were carried out on multiple dates throughout March 2016. The search strategy was employed to determine the extent of social media usage by foundations, organizations, societies, support groups, medical centers, and journals dedicated to the care of patients with hydrocephalus.

\section{Facebook}

Accounts related to "hydrocephalus" were organized by "pages" only and "groups" only. Each account was manually screened, and pages with more than 100 likes or groups with more than 10 members were included. Accounts related to animal hydrocephalus were excluded. Publicly accessible social media metrics were compiled from each account, including the number of "likes" for Facebook pages, the number of members in Facebook groups, the year the page or group was created, the privacy status, and the account's country of origin. Most of the metrics were unavailable for data collection from "private" groups, as Facebook restricts access to information to members within these groups. The number of users within the private group, however, is publicly accessible.

\section{YouTube}

The search results were filtered by YouTube "channels" only and YouTube "videos" only. Each channel account was manually screened, and only accounts created by foundations, organizations, societies, support groups, medical centers, and journals were included. Channels with no subscribers and channels with an empty "about" page were excluded from data collection. All YouTube videos related to hydrocephalus (excluding animal hydrocephalus) with more than 500 views were included in the study. Social media metrics were compiled from each channel and video, including the views count, the number of subscribers, the country of origin, the number of likes, the number of comments, the date and year of creation, and the type of video (e.g., patient education or surgical videos).

\footnotetext{
Twitter

Using the term "hydrocephalus," we searched Twitter for accounts "from everywhere" and "from everyone"
}

to maximize search results. Each account was manually screened, and only accounts created by foundations, organizations, societies, support groups, medical centers, and journals were included. Inaccessible accounts, accounts with no activity, and accounts not related to health care (e.g., a music band called "Hydrocephalus") were excluded from data collection. Metrics were compiled from each Twitter account, including the number of "followers," the number of tweets, the number of Twitter likes, the year of creation, and the country of origin.

\section{Data Categorization}

A categorization scheme was employed to classify the purpose of pages, groups, accounts, channels, and videos on each of the 3 SMNs. This scheme was developed following the screening of 100 titles for accounts and videos. ${ }^{3}$ In particular, data were categorized based on public titles, site biography, or user-generated account descriptions. Titles were independently classified by 2 authors (G.A.E. and N.M.A.), and agreement was assessed with Cohen's kappa.

\section{Statistical Analysis}

Descriptive and summary statistics, including the mean, median, standard deviation, and interquartile range (IQR), were calculated for all social media metrics analyzed. Since social media metrics are not normally distributed,, 311 we employed nonparametric tests for significance (MannWhitney U-test for 2 groups or Kruskal-Wallis test for multiple comparisons); $\mathrm{p}$ values below 0.05 were deemed statistically significant. Descriptive statistics alone are presented for SMN pages with insufficient sample sizes for statistical comparison (Twitter accounts and YouTube channels). All statistical analyses were performed using IBM SPSS Statistics version 21 (IBM Corp.).

\section{Ethical Considerations}

Data were exclusively collected from publicly accessible SMN accounts. Usernames were not recorded, and no interactions were made with users on the SMNs. In accordance with the exclusion criteria of the Canadian TriCouncil Policy Statement for research studies, institutional research ethics board approval was not required, as all data collected are publicly available.

\section{Results}

Our search identified 30 Facebook pages, 213 Facebook groups, 17 Twitter accounts, 10 YouTube channels, and 253 YouTube videos. Descriptive and comparative statistics for accounts and videos related to hydrocephalus on 3 major SMNs are summarized in Table 1 . Table 2 summarizes the top 5 accounts related to hydrocephalus on the various SMNs. Almost all accounts and videos were dedicated to pediatric hydrocephalus (94\%), reflecting a strong emphasis on children and their families. The earliest accounts were created in 2007, and the number of accounts created per year peaked in 2011, with 65 new accounts (Fig. 1). Based on title screening, there were 2 categories of accounts (foundations and patient support groups) seen 
TABLE 1. Accounts, users, and views related to hydrocephalus on SMNs

\begin{tabular}{lccc}
\hline \multicolumn{1}{c}{ SMN } & $\begin{array}{c}\text { Accounts/ } \\
\text { Videos (no.) }\end{array}$ & Users or Views* & p Value \\
\hline Facebook & & & $<0.0001$ \\
\hline Page & 30 & $553(306-1646)$ & \\
\hline Group & 213 & $82(35-211)$ & \\
\hline Facebook groups & & & 0.0005 \\
\hline Public & 59 & $44(26-139)$ & \\
\hline$\quad$ Private & 154 & $95(40-218)$ & NA \\
\hline $\begin{array}{l}\text { Twitter } \\
\text { YouTube channels }\end{array}$ & 17 & $240(75-943)$ & NA \\
\hline $\begin{array}{l}\text { YouTube videos } \\
\text { Medical/surgical } \\
\text { products }\end{array}$ & 15 & $10,895(986-33,372)$ & \\
\hline $\begin{array}{c}\text { Pts' experience \& } \\
\text { support }\end{array}$ & 149 & $2029(995-5816)$ & \\
\hline $\begin{array}{l}\text { Tx procedures } \\
\text { Educational }\end{array}$ & 22 & $3211(1332-29,635)$ & \\
\hline
\end{tabular}

$\mathrm{NA}=$ not applicable; pts $=$ patients; $\mathrm{Tx}=$ treatment.

* Social media metrics (number of users and video views) are presented as medians (IQR).

among Facebook, Twitter, and YouTube channels; and 4 categories among YouTube videos (medical/surgical products for shunt valves mainly, patients' experience and support following treatment, treatment procedure details and techniques, and educational videos on clinical presentation and pathophysiology). Cohen's kappa was 0.82 , representing good interrater agreement for the categories.

\section{Facebook}

A total of 243 hydrocephalus-related Facebook accounts were identified. Slightly more than half of the accounts were created in the US (53\%); $8 \%$ were created in the United Kingdom(UK), 5\% in Canada, $4 \%$ in India, and $3 \%$ in Germany. The Facebook community had a total of 122,554 users in pages and groups (range 4-24,496, median 105, IQR 37-273). The majority of users were on Facebook pages $(\mathrm{p}<0.0001)$. Of the 213 hydrocephalus-related groups, 59 were open to the public, and 154 were private. The median number of users was significantly greater in private groups than in public groups $(\mathrm{p}=0.0005)$. Posts on private groups are only visible to members within the group. Based on title screenings, Facebook accounts were predominately created to provide support for caregivers and patients with hydrocephalus.

\section{Twitter}

Seventeen hydrocephalus-related Twitter accounts were identified. These Twitter accounts had a total of 28,282 users or "followers" (range 5-16,300, median 240, IQR 75-943). Eight accounts were created by nonprofit hydrocephalus organizations, such as ShineUK and the Hydrocephalus Association. Six accounts were created for fund-raising, such as Hydrocephalus Walk and Winners Wear Yellow.

\section{YouTube}

We identified 10 hydrocephalus-related YouTube channels with 1240 subscribers and a total of 300,243 views in our results. The majority of subscribers $(36 \%)$ and views (43\%) belonged to 1 channel (Hydrocephalus Association). More than 250 YouTube videos on the topic of hydrocephalus were also identified. The total number of views was 2,842,467 (range 502-227,924, median 2372, IQR 1267-7659). The majority of YouTube videos (59\%) were created to share patients' experience and to provide support to others with hydrocephalus. There was no statistically significant difference between the number of views in the 4 different categories $(p=0.26)$ (Table 1$)$.

\section{Discussion}

Social support has been shown to be beneficial for patients and caregivers. ${ }^{6,10}$ SMNs are commonly used by patients with chronic disease as important sources of information, support, and engagement. ${ }^{5,13,16,22,23}$ They are widely available and easily accessible, with approximately $65 \%$ of the American population having active social media accounts. ${ }^{21}$ Facebook, YouTube, and Twitter are popular SMNs; of these, Facebook is the most popular, with 1.7 billion active users per month (Facebook company information, http://newsroom.fb.com/company-info/). Many disease-specific groups or communities exist on SMNs. Extensive studies have been published exploring the characteristics of and the dynamics within those communities, including social media analyses on multiple sclerosis, ${ }^{22}$ dementia, ${ }^{23}$ autism, ${ }^{16}$ breast cancer, ${ }^{5}$ diabetes mellitus, ${ }^{13}$ hypertension, ${ }^{1}$ and obesity. ${ }^{8}$ This study provides the first analysis regarding the topic of hydrocephalus on 3 major SMNs: Facebook, YouTube and Twitter.

Parents of children with shunt-treated hydrocephalus frequently use the internet and social media for support and information gathering. Eighty percent of parents surveyed $(n=300)$ in the neurosurgical clinics at the University of Alabama used the internet to acquire hydrocephalus-related information. ${ }^{17}$ There was significantly less access by caregivers of minority race, lower income level, and lower education level. More than half of caregivers of children with hydrocephalus used SMNs for support and hydrocephalus-related information, with Facebook and YouTube being the preferred social media platforms. ${ }^{17}$ In this study, we have provided descriptive and comparative statistics for accounts and videos related to hydrocephalus on Facebook, YouTube, and Twitter. Of the 3 SMNs, we found that Facebook was the most popular, with over 120,000 users in pages and groups. This finding is consistent with the findings of a national survey that has identified Facebook as the most popular social media site. ${ }^{9}$ We have also shown that the earliest accounts from all 3 SMNs related to hydrocephalus were created in 2007, and account creation peaked in 2011, declining thereafter. This pattern likely represents an initially growing online hydrocephalus community that is now stabilizing. For instance, new Facebook users seeking support or information related to hydrocephalus may choose to join existing pages and/or groups instead of creating new ones. It is important to note that we were not able to identify 
TABLE 2. Top 5 accounts related to hydrocephalus on various SMNs

\begin{tabular}{|c|c|c|c|}
\hline Platform \& Account & Users, Views, or Followers* & Country & Year \\
\hline \multicolumn{4}{|l|}{ Facebook page } \\
\hline Hydrocephalus Association & 24,496 & US & 2009 \\
\hline SHINE Charity & 12,308 & UK & 2011 \\
\hline Pediatric Hydrocephalus Foundation, Inc. & 8502 & US & 2009 \\
\hline International Federation for Spina Bifida and Hydrocephalus & 3677 & Belgium & 2008 \\
\hline Hydrocephalus Treatment Surgery India & 2755 & India & 2014 \\
\hline \multicolumn{4}{|l|}{ Facebook group } \\
\hline Spina Bifida \& Hydrocephalus Parenting Support Group & 1972 & UK & 2015 \\
\hline Hydrocephalus Foundation of the Philippines & 1855 & Philippines & 2015 \\
\hline UK Hydrocephalus Support Network & 1306 & UK & 2015 \\
\hline Hydrocephalus Awareness of Columbus County & 655 & US & 2015 \\
\hline Hydrocephalus Awareness Pageant & 553 & US & 2015 \\
\hline \multicolumn{4}{|l|}{ YouTube channel } \\
\hline Hydrocephalus Association & 130,097 & US & 2010 \\
\hline CURE International & 91,636 & Worldwide & 2008 \\
\hline SHINE Charity & 39,817 & UK & 2011 \\
\hline Association for Spina Bifida and Hydrocephalus & 14,037 & NA & 2010 \\
\hline International Federation for Spina Bifida and Hydrocephalus & 11,376 & Belgium & 2010 \\
\hline \multicolumn{4}{|l|}{ Twitter } \\
\hline SHINE UK Charity & 16,300 & UK & 2010 \\
\hline Hydrocephalus Association & 4224 & US & 2009 \\
\hline Winners Wear Yellow† & 3365 & UK & 2011 \\
\hline International Federation for Spina Bifida and Hydrocephalus & 1429 & Belgium & 2009 \\
\hline Virtual Balloon Races† & 943 & UK & 2012 \\
\hline
\end{tabular}

the year of creation for 153 private Facebook groups, as Facebook restricts access to information in private groups. Those groups could have been created in different years, and this would change the number and overall pattern of new accounts created per year.

One of the recurrent limitations of social media for health communication explored in several studies is the

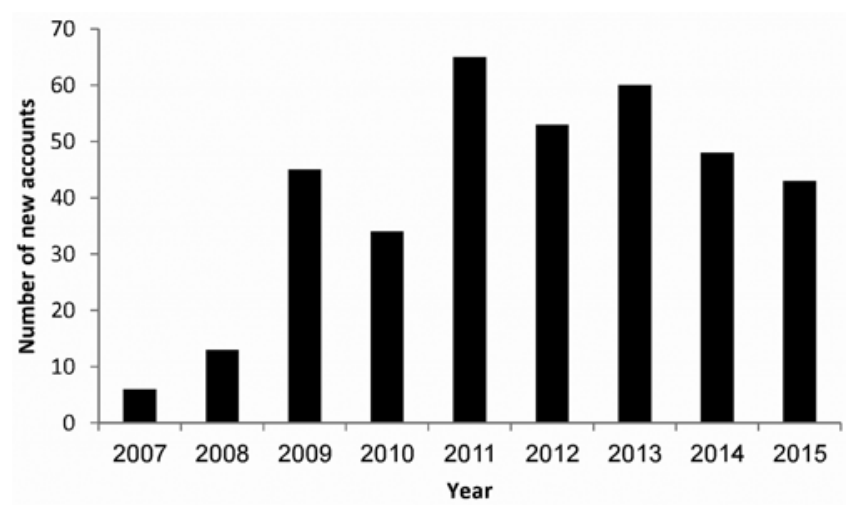

FIG. 1. Number of new hydrocephalus-related social media accounts created on Facebook, Twitter, and YouTube annually from 2007 through 2015. lack of confidentiality and privacy. ${ }^{12,15,18}$ Interestingly, most of the hydrocephalus-related Facebook groups were private, and there was a significant difference in user "medians" between private groups and public groups (95 vs 44). These findings illustrate a tendency among users to sustain privacy in their communications on Facebook. Although limiting access to a Facebook group does not guarantee quality or reliability of the health information shared within the group, it is a step in the right direction to reduce inaccurate health information and prevent exploitation of shared personal stories. These findings may explain why Twitter was not a popular platform for hydrocephalus-related use, with only 17 accounts, as it does not offer private group discussions. Furthermore, for patients and caregivers, Facebook is a convenient platform to share personal stories, with its limits of 8000 characters for "comments" and 5000 characters for "wall posts." Twitter, on the other hand, only allows 140 characters per message.

Our analysis revealed 253 hydrocephalus-related YouTube videos with a total of 2.8 million views. The majority of posted videos were about patients' experience and support, with 149 videos and 1.3 million views in total. Videos about hydrocephalus treatment procedures and surgical products were also popular, with over 670,000 views in total, and had the highest median number of views. 
Patients with hydrocephalus or parents of children with hydrocephalus might be using YouTube videos as a means of preparing for their upcoming hospital experience. In a study exploring the utility of YouTube videos in providing a "virtual hospital experience" for patients undergoing hip and knee replacement procedures, results showed a trend toward less anxiety in patients who viewed the YouTube videos. ${ }^{19}$ Incorporating YouTube videos might be useful for optimizing preoperative consultations and counseling for patients with hydrocephalus and their caregivers.

\section{Strengths, Limitations, and Future Directions}

For this study, data were acquired from 3 of the most popular social networking sites to provide a description of social media use for the topic of hydrocephalus. Previous works on the utility of SMNs in chronic disease were focused on only one of these sites..$^{1,5,13,16,22,23}$ Several limitations of this study should be noted. The term "hydrocephalus" likely underestimates the size of the online hydrocephalus community, as patients or caregivers may also use terms such as "shunt." The latter is more generalized and a preliminary search using "shunt" included numerous unrelated accounts pertaining to liver shunts and even musical groups. It should be also noted that due to privacy polices we were not able to identify individual Facebook users, YouTube viewers, and Twitter followers and were therefore unable to analyze the degree of user overlap among groups and platforms, and this may magnify our sample size.

Only publicly available information was used in our analysis. Most of the information about private Facebook groups, including country of origin, year of establishment, engagement, and whether these groups were deleted or removed, was inaccessible. This limited the comparison between public and private Facebook groups to the number of users in each category. Facebook page or group titles, Twitter account titles, and YouTube video titles were largely used to discern the purpose of use. This may not be accurate, as 1 page or group might be used for multiple reasons, such as providing emotional support and fundraising at the same time. This study does not provide an analysis of content made by users on SMNs or show how SMNs can influence patient referrals for hydrocephalus. Future research is needed to provide a qualitative description of hydrocephalus-related posts, and a prospective study surveying new hydrocephalus patients at care centers would better elucidate the different sources of referrals. Finally, social media statistics are dynamic, with changing memberships and content that can be altered by account owners or third-party software products.

\section{Conclusions}

In this study, we provide a point prevalence description and analysis of social media use related to hydrocephalus on 3 popular social networks. Users tend to seek social support and privacy in their online communications, and they are interested in learning the details of treatment procedures. Findings in this report will inform practicing physicians of the presence and magnitude of the online community interested in hydrocephalus, and serve as a resource for patients interested in seeking relevant social support.

\section{References}

1. Al Mamun M, Ibrahim HM, Turin TC: Social media in communicating health information: an analysis of Facebook groups related to hypertension. Prev Chronic Dis 12:E11, 2015

2. Alotaibi NM, Badhiwala JH, Nassiri F, Guha D, Ibrahim GM, Shamji MF, et al: The current use of social media in neurosurgery. World Neurosurg 88:619-624, 624.e1-624. e7, 2016

3. Alotaibi NM, Samuel N, Wang J, Ahuja CS, Guha D, Ibrahim GM, et al: The use of social media communications in brain aneurysms and subarachnoid hemorrhage: a mixedmethod analysis. World Neurosurg 98:456-462, 2017

4. Barakat LP, Linney JA: Children with physical handicaps and their mothers: the interrelation of social support, maternal adjustment, and child adjustment. J Pediatr Psychol 17:725-739, 1992

5. Bender JL, Jimenez-Marroquin MC, Jadad AR: Seeking support on Facebook: a content analysis of breast cancer groups. J Med Internet Res 13:e16, 2011

6. Capuzzi C: Maternal attachment to handicapped infants and the relationship to social support. Res Nurs Health 12:161167,1989

7. Casey AT, Kimmings EJ, Kleinlugtebeld AD, Taylor WA, Harkness WF, Hayward RD: The long-term outlook for hydrocephalus in childhood. A ten-year cohort study of 155 patients. Pediatr Neurosurg 27:63-70, 1997

8. Chou WY, Prestin A, Kunath S: Obesity in social media: a mixed methods analysis. Transl Behav Med 4:314-323, 2014

9. Duggan M: Mobile messaging and social media - 2015. Pew Research Center. August 19, 2015. (http://www.pewinternet. org/2015/08/19/mobile-messaging-and-social-media-2015/) [Accessed March 22, 2017]

10. Dunst CJ, Trivette CM, Cross AH: Mediating influences of social support: personal, family, and child outcomes. Am J Ment Defic 90:403-417, 1986

11. Eysenbach G: Can tweets predict citations? Metrics of social impact based on Twitter and correlation with traditional metrics of scientific impact. J Med Internet Res 13:e123, 2011

12. Fernandez-Luque L, Elahi N, Grajales FJ III: An analysis of personal medical information disclosed in YouTube videos created by patients with multiple sclerosis. Stud Health Technol Inform 150:292-296, 2009

13. Greene JA, Choudhry NK, Kilabuk E, Shrank WH: Online social networking by patients with diabetes: a qualitative evaluation of communication with Facebook. J Gen Intern Med 26:287-292, 2011

14. Kulkarni AV, Cochrane DD, McNeely PD, Shams I: Medical, social, and economic factors associated with health-related quality of life in Canadian children with hydrocephalus. J Pediatr 153:689-695, 2008

15. Moen A, Smørdal O, Sem I: Web-based resources for peer support-opportunities and challenges. Stud Health Technol Inform 150:302-306, 2009

16. Mohd Roffeei SH, Abdullah N, Basar SK: Seeking social support on Facebook for children with autism spectrum disorders (ASDs). Int J Med Inform 84:375-385, 2015

17. Naftel RP, Safiano NA, Falola MI, Shannon CN, Wellons JC III, Johnston JM Jr: Technology preferences among caregivers of children with hydrocephalus. J Neurosurg Pediatr 11:26-36, 2013

18. Nordqvist C, Hanberger L, Timpka T, Nordfeldt S: Health professionals' attitudes towards using a Web 2.0 portal for 
child and adolescent diabetes care: qualitative study. J Med Internet Res 11:e12, 2009

19. O’Connor MI, Brennan K, Kazmerchak S, Pratt J: YouTube videos to create a "virtual hospital experience" for hip and knee replacement patients to decrease preoperative anxiety: a randomized trial. Interact J Med Res 5:e10, 2016

20. Patel R, Chang T, Greysen SR, Chopra V: Social media use in chronic disease: a systematic review and novel taxonomy. Am J Med 128:1335-1350, 2015

21. Perrin A: Social media usage: 2005-2015. Pew Research Center. October 8, 2015. (http://www.pewinternet. org/2015/10/08/social-networking-usage-2005-2015/) [Accessed March 22, 2017]

22. Ramagopalan S, Wasiak R, Cox AP: Using Twitter to investigate opinions about multiple sclerosis treatments: a descriptive, exploratory study. F1000 Res 3:216, 2014

23. Robillard JM, Johnson TW, Hennessey C, Beattie BL, Illes J: Aging 2.0: health information about dementia on Twitter. PLoS One 8:e69861, 2013

\section{Disclosures}

Dr. Alotaibi serves as social media coordinator for the Journal of Neurosurgery Publishing Group (JNSPG). JNSPG had no role in study design, data collection and analysis, decision to publish, or preparation of the manuscript for submission.

\section{Author Contributions}

Conception and design: Alotaibi. Acquisition of data: Elkarim, Alotaibi. Analysis and interpretation of data: all authors. Drafting the article: Elkarim, Alotaibi, Samuel, Wang. Critically revising the article: Kulkarni, Ibrahim, Fallah, Weil. Reviewed submitted version of manuscript: Kulkarni, Elkarim, Samuel, Wang, Ibrahim, Fallah, Weil. Approved the final version of the manuscript on behalf of all authors: Kulkarni. Statistical analysis: Alotaibi. Study supervision: Kulkarni, Alotaibi.

\section{Supplemental Information}

\section{Previous Presentations}

Portions of this manuscript were presented as part of the oral abstract platform at the 45th Annual Meeting of the AANS/ CNS Joint Section on Pediatric Neurosurgery, Orlando, Florida, December 5-8, 2016.

\section{Correspondence}

Abhaya V. Kulkarni, Division of Neurosurgery, Hospital for Sick Children, 555 University Ave., Rm. 1503, Toronto, ON M5G 1X8, Canada.email: abhaya.kulkarni@sickkids.ca. 